\title{
Updating of an Occluded Moving Target for Interceptive Saccades
}

\author{
Joost C. Dessing \\ Centre for Vision Research, York University, Toronto, Ontario M3J 1P3, Canada \\ Review of Fleuriet and Goffart
}

In daily life, many objects of interest move relative to our bodies. Adequate perception of such objects involves keeping them foveated through coordinated saccadic and pursuit eye movements. If an object moves in the visual periphery it can only be pursued after an interceptive saccade, which brings the object's image onto the fovea. These interceptive saccades are generally accurate, even if they occur during occlusion (Bennett and Barnes, 2006). This indicates that the brain accounts for the continuous displacement of a moving target. Although much is known about the brain mechanisms underlying eye movements, the specifics of how the brain achieves accurate interceptive saccades are still debated.

One of these debates centers on how the oculomotor system accounts for target displacement resulting from motion when planning saccades. According to one view, the saccade generator incorporates two distinct drives: a position signal within the superior colliculus (SC) relaying target position, and an extracolicular motionbased signal accounting for subsequent target displacement (Keller et al., 1996; Optican and Quaia, 2002; Guan et al., 2005; Cassanello et al., 2008). These signals are postulated to converge on premotor neu-

Received March 10, 2012; revised April 9, 2012; accepted April 11, 2012. I thank Robert Marino for discussions on this paper.

Correspondence should be addressed to Joost C. Dessing, Centre for Vision Research, York University, 4700 Keele Street, Toronto, 0N M3J 1P3, Canada. E-mail: joost@yorku.ca.

DOI:10.1523/JNEUROSCI.1193-12.2012

Copyright $\odot 2012$ the authors $\quad$ 0270-6474/12/327767-02\$15.00/0 rons in the brainstem. This "dual-drive" scheme was proposed based on the observation that SC activity during interceptive saccades does not reflect target position at saccade end and that the kinematics of interceptive saccades differ from those of saccades to stationary targets (Keller et al., 1996; Guan et al., 2005). An alternative view suggests that motion compensation occurs within or upstream to the SC, which keeps track of the expected target position (Fleuriet et al., 2011). In this "remapping" scheme, saccades to stationary and moving targets could then be accomplished using the same feedback mechanism. A motionbased prediction of target displacement has in fact been observed in activity of the frontal eye fields (FEF) (Xiao et al., 2007; Cassanello et al., 2008; Ferrera and Barborica, 2010), which projects to SC. In barn owls, activity within the auditory map in the tectum (the homolog of SC in monkeys) also codes a position ahead of the moving sound that gave rise to the activity (Witten et al., 2006).

A recent study published in The Journal of Neuroscience (Fleuriet and Goffart, 2012) addressed the distinction between these schemes on the basis of experiments involving microstimulation of the SC. Monkeys made interceptive saccades to, and subsequently pursued, moving targets; these targets were occluded during the interceptive saccades. In some trials, eye position was perturbed at occlusion onset through microstimulation in deep SC. Fleuriet and Goffart (2012) showed that, even when the saccades started from a different position than saccades without microstimulation, they always landed near the (occluded) target. Moreover, the subsequent interceptive saccade often adequately compensated for the different amounts of invisible target motion occurring during additional delays induced by the microstimulation. Importantly, this fascinating finding argues against the use of a fixed prediction of future target position. Rather, it suggests dynamic updating of the saccade plan to account for microstimulation-induced changes in eye position and ongoing changes in target position regardless of target visibility.

In discussing these findings, Fleuriet and Goffart (2012) identified several problems with the dual-drive scheme. Because of these concerns, they suggested that the ability to make accurate interceptive saccades is better explained by the alternative remapping scheme. Among other issues, they questioned the notion of a "positional snapshot" of where the target was initially detected, which they see as fundamental to the dual-drive hypothesis. In addition, they questioned whether the target's future displacement could be accurately calculated given that the time interval for this integration cannot be considered constant. A saccadic position error signal that depends on target position at a certain time before saccade onset, however, does not necessarily require a discrete snapshot; it could just be the last target position to influence SC activity before saccade onset, not unlike in the remapping scheme. Accordingly, the oculomotor system must account 
for target displacement between final SC read-out and saccade end, which is a more predictable interval. The only difference between this scheme and the remapping scheme advocated by Fleuriet and Goffart (2012) is the stage at which target motion compensation occurs. In both schemes, interceptive saccades only land at the correct (occluded) target position if SC activity is updated for the microstimulation-induced change in eye position, just like for stationary targets (Sparks and Mays, 1983). Updating for target displacement during the additional delay would be automatic if continuous (cortical) input to SC keeps track of retinal target position, even during periods of occlusion (Xiao et al., 2007; Ferrera and Barborica, 2010). Thus, the results clearly demonstrate continuous updating of target position, but do show whether motion compensation occurs within (inputs to) SC or downstream of SC.

The similarity between the aforementioned control schemes underscores the difficulty with inferring such schemes from kinematics alone (Beek et al., 2003). The currently available physiological and anatomical data concerning the origin of target motion compensation signals and the route by which these influence the saccade plan also provide no definitive conclusion. The stream is thought to predominantly involve medial temporal motion-sensitive area (Groh et al., 1997) and FEF (Xiao et al., 2007; Cassanello et al., 2008; Ferrera and Barborica, 2010), or possibly the cerebellum (Optican and Quaia, 2002). According to the remapping hypothesis, motion compensation occurs through inputs from these areas to SC (Fleuriet et al., 2011); the dual-drive hypothesis holds that this compensation occurs through similar inputs to brainstem premotor centers. In terms of gross anatomical connections, both hypotheses are possible, and are indeed not mutually exclusive.

The critical data, thus, concerns SC activity for interceptive saccades. The remapping hypothesis suggests SC codes the expected target position, which includes motion compensation; the dual-drive hypothesis suggests SC codes a target position a short interval in the past, not including motion compensation. Keller et al.'s (1996) finding that activity in the deep SC does not code the end position of an interceptive saccade is insufficient to assess whether motion compensation occurs within SC. More recent work sug- gests that the SC (i.e., tectum) might code the future position of a moving target i.e., performs motion compensation (Witten et al., 2006), but this concerned a different species and different sensory modality. I am unaware of more detailed data concerning visual SC activity for interceptive saccades, which would be needed to definitively distinguish between the remapping and dualdrive hypotheses, in terms of the proposed route for motion compensation.

The saccades observed by Fleuriet and Goffart (2012) were influenced by the target trajectory in several ways. Inaccuracies of interceptive saccades were observed for targets initially moving in the direction of the induced saccade; these also depended on the vector encoded at the stimulation site (Fleuriet and Goffart, 2012, their Fig. 8). Another effect of the target trajectory can be appreciated from Fleuriet and Goffart's Figures 2, 6, and 7: the microstimulation-induced saccades appear to reflect a vector-average between the saccade coded at the stimulation site and position-dependent aspects of the target trajectory (Edelman and Keller, 1998; Katnani and Gandhi, 2011; Nummela and Krauzlis, 2011). Fleuriet and Goffart (2012) interpreted the inaccuracies to suggest that microstimulation interferes with processing of target motion or expected target position. However, because their paradigm involved a single target velocity and fixed preocclusion interval per block, any expected target position (including motion compensation) strongly correlated with target position at occlusion (not including motion compensation). This is relevant with respect to the distinction between the two schemes discussed above and highlights the need for more in-depth investigations of this paradigm in which more target trajectories are randomly interspersed (i.e., multiple initial target positions, velocities, and preocclusion windows).

Fleuriet and Goffart's (2012) examination of interceptive saccades following microstimulation of SC yielded the fascinating finding that the saccade plan can be corrected for the induced changes in eye position, as well as the target displacement occurring during induced delays. These findings leave the question of where exactly target motion compensation within the oculomotor system, which sets apart the dual-drive and remapping schemes for interceptive saccades. As discussed,
Fleuriet and Goffart's (2012) findings thus motivate further research that would affords a definitive dissociation of these two schemes.

\section{References}

Beek PJ, Dessing JC, Peper CE, Bullock D (2003) Modelling the control of interceptive actions. Philos Trans R Soc Lond B Biol Sci 358:1511-1523.

Bennett SJ, Barnes GR (2006) Combined smooth and saccadic ocular pursuit during the transient occlusion of a moving visual object. Exp Brain Res 168:313-321.

Cassanello CR, Nihalani AT, Ferrera VP (2008) Neuronal responses to moving targets in monkey frontal eye fields. J Neurophysiol 100:1544-1556.

Edelman JA, Keller EL (1998) Dependence on target configuration of express saccade-related activity in the primate superior colliculus. J Neurophysiol 80:1407-1426.

Ferrera VP, Barborica A (2010) Internally generated error signals in monkey frontal eye field during an inferred motion task. J Neurosci 30:11612-11623.

Fleuriet J, Goffart L (2012) Saccadic interception of a moving visual target after a spatiotemporal perturbation. J Neurosci 32:452-461.

Fleuriet J, Hugues S, Perrinet L, Goffart L (2011) Saccadic foveation of a moving visual target in the rhesus monkey. J Neurophysiol 105:883-895.

Groh JM, Born RT, Newsome WT (1997) How is a sensory map read out? Effects of microstimulation in visual area MT on saccades and smooth pursuit eye movements. J Neurosci 17:4312-4330.

Guan Y, Eggert T, Bayer O, Büttner U (2005) Saccades to stationary and moving targets differ in the monkey. Exp Brain Res 161:220-232.

Katnani HA, Gandhi NJ (2011) Order of operations for decoding superior colliculus activity for saccade generation. J Neurophysiol 106: $1250-1259$.

Keller EL, Gandhi NJ, Weir PT (1996) Discharge of superior collicular neurons during saccades made to moving targets. J Neurophysiol 76:3573-3577.

Nummela SU, Krauzlis RJ (2011) Superior colliculus inactivation alters the weighted integration of visual stimuli. J Neurosci 31:8059-8066.

Optican LM, Quaia C (2002) Distributed model of collicular and cerebellar function during saccades. Ann N Y Acad Sci 956:164-177.

Sparks DL, Mays LE (1983) Spatial localization of saccade targets. I. Compensation for stimulation-induced perturbations in eye position. J Neurophysiol 49:45-63.

Witten IB, Bergan JF, Knudsen EI (2006) Dynamic shifts in the owl's auditory space map predict moving sound location. Nat Neurosci 9:1439-1445.

Xiao Q, Barborica A, Ferrera VP (2007) Modulation of visual responses in macaque frontal eye field during covert tracking of invisible targets. Cereb Cortex 17:918-928. 